Quim. Nova, Vol. 35, No. 5, 1020-1024, 2012

\title{
QUANTIFICAÇÃO DOS ISÔMEROS ÁCIDO L-ASCÓRBICO E ÁCIDO D-ISO-ASCÓRBICO EM GELEIAS DE FRUTAS POR CROMATOGRAFIA LÍQUIDA DE ALTA EFICIÊNCIA
}

\author{
Raquel Grando de Oliveira*, Helena Teixeira Godoy e Marcelo Alexandre Prado \\ Departamento de Ciência de Alimentos, Faculdade de Engenharia de Alimentos, Universidade de Campinas, CP 6121, 13083-862 \\ Campinas - SP, Brasil
}

Recebido em 19/7/11; aceito em 2/12/11; publicado na web em 31/1/12

\begin{abstract}
QUANTIFICATION OF L-ASCORBIC ACID AND D-ISO-ASCORBIC ACID IN JELLIES FRUIT BY HIGH PERFORMANCE LIQUID CHROMATOGRAPHY. Ascorbic acid has important nutritional characteristics such as high antioxidant potential, preventing diverse damage and diseases in the tissues and the process of aging. Different isomeric forms of the ascorbic acid can be found in nature and each one have different potential antioxidant and different activity pro-vitamin C. This work examined a method to detect and quantify the isomers L-ascorbic acid (LAA) and D-iso-ascorbic acid (DIAA) in jelly fruit. The method showed acceptable selectivity, linearity, repeatability and recovery. DIAA was not found in the analyzed samples, but LAA was found up to $605 \mathrm{mg}$ in $100 \mathrm{~g}$ of sample.
\end{abstract}

Keywords: ascorbic acid; iso-ascorbic acid; fruit jelly.

\section{INTRODUÇÃO}

Ácido ascórbico é um composto com importantes características nutricionais. Antigamente, este composto era conhecido por sua capacidade de prevenir o escorbuto, ${ }^{1}$ mas atualmente existe grande interesse científico na sua capacidade antioxidante e na sua funcionalidade nutricional para o organismo humano. ${ }^{2} \mathrm{~A}$ atividade antioxidante pode atuar capturando radicais livres tóxicos e espécies reativas de oxigênio, prevenindo algumas doenças e disfunções nos tecidos e reduzindo o processo de envelhecimento. ${ }^{3,4} \mathrm{~A}$ vitamina $\mathrm{C}$ pode atuar ainda na formação do tecido conjuntivo e no transporte de íons. ${ }^{1}$ Casos de toxicidade envolvendo sua ingestão são raros, pois se trata de uma vitamina hidrossolúvel e é regularmente excretada pelo organismo, no entanto, doses excessivas já foram relacionadas a cálculos renais e, em casos mais raros, com anemia, causada pela interferência na absorção de vitamina B12. ${ }^{5}$

Mais de $90 \%$ da vitamina C presente na dieta humana é proveniente de frutas e vegetais, ${ }^{6}$ principalmente em frutas cítricas como laranjas e seus sucos. ${ }^{7} \mathrm{O}$ teor pode variar significantemente de acordo com as condições de cultivo, a época de plantio, a incidência solar, o estágio de maturação, o manuseio pós-colheita e qualidade do solo. ${ }^{8}$

Seu uso é frequente nas indústrias alimentícia e farmacêutica, sendo estimado em 154 milhões de toneladas em 2007. Aproximadamente $50 \%$ da produção são destinados à adição em alimentos com a intenção de prevenir a degradação de pigmentos, evitar o escurecimento enzimático, diminuir perdas de sabor e aroma, proteger e aumentar fatores nutricionais e, ainda aumentar, a vida de prateleira. ${ }^{2}$

Em meio seco, o ácido ascórbico é estável, mas pode degradar gradualmente em função da exposição à luz. ${ }^{9}$ Em solução, este composto é facilmente oxidado e degradado, sendo o processo acelerado em presença de cobre, ferro e álcali. ${ }^{10}$ Devido à instabilidade, é necessário que as etapas de extração, detecção e quantificação sejam feitas sob a observação de condições controladas de temperatura e $\mathrm{pH}$, ausência de oxigênio e metais e presença de agentes estabilizantes. ${ }^{8,11,12}$

Existem vários métodos capazes de determinar ácido ascórbico em amostras. ${ }^{4}$ Os métodos biológicos foram os primeiros desenvolvidos e se baseiam na quantidade necessária para prevenir o escorbuto

\footnotetext{
*e-mail: rgrando@gmail.com
}

em cobaias. Estes não são mais frequentemente aplicados, devido ao elevado tempo de análise, alto custo e baixa repetitividade. ${ }^{9,13}$ Atualmente os métodos químicos são os mais utilizados. ${ }^{9}$

Tilmans, citado por Aldrigue, ${ }^{9}$ foi o responsável por desenvolver um método colorimétrico muito empregado nos dias de hoje. Após aperfeiçoamentos, este método foi considerado como padrão para a determinação de sucos e preparados, de acordo com a Associação Oficial de Química Analítica (AOAC). ${ }^{14,15} \mathrm{O}$ método corresponde a uma titulação com o indicador 2,6-diclorofenol-indofenol (DCIP), na qual o ácido ascórbico reduz este reagente inicialmente azul a uma solução incolor. No ponto final da titulação, o excesso de indicador não reduzido confere à solução uma coloração rosada, portanto, o ponto final pode ser verificado visualmente, em adição com métodos eletroquímicos e fotométricos. ${ }^{9}$ Esta é uma técnica de fácil aplicação e baixo custo, principalmente quando comparada com técnicas cromatográficas, por exemplo. O uso do DCIP é frequente, tanto para produtos alimentícios quanto para farmacêuticos, sendo ainda usado como base de comparação para novos métodos.

Apesar da AOAC indicar este método como oficial, não pode ser aplicado para todas as matrizes. Substâncias presentes naturalmente em frutas como taninos, compostos sulfidrílicos e metais como cobre, ferro e cobalto são também oxidados por DCIP. ${ }^{15}$ Outro empecilho está no fato da coloração natural das amostras interferir na visualização do ponto final.

O ácido ascórbico apresenta uma estrutura com seis carbonos e pode ser encontrado em algumas formas isoméricas: ácido L-ascórbico (LAA), ácido D-ascórbico (DAA), ácido L-iso-ascórbico (LIAA) e ácido D-iso-ascórbico (DIAA) (Figura 1). O isômero mais encontrado naturalmente em frutas é o LAA, que também é o mais utilizado no organismo para as funções biológicas. ${ }^{16}$ DIAA, também conhecido como ácido eritrórbico e ácido D-araboascórbico apresenta propriedade antioxidante similar ao LAA, mas apenas $5 \%$ de sua atividade pró-vitamínica C. Este fato está associado à diferença estrutural na posição do grupo hidroxila no carbono $5 .{ }^{17-19}$ DIAA não é normalmente encontrado em alimentos naturais, mas apresenta grande importância na tecnologia alimentar, podendo ser adicionado como antioxidante em bebidas, vinhos e carne, substituindo o LAA, com ganhos econômicos, sem que a capacidade antioxidante seja perdida. $^{20,21}$ 


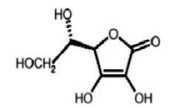

Ácido L-ascórbico

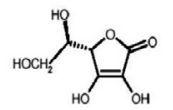

Ácido D-ascórbico
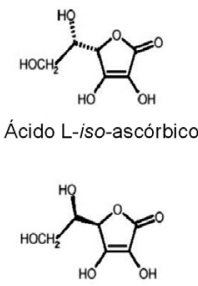

Ácido D-iso-ascórbico
Figura 1. Formas isoméricas do ácido ascórbico

Vários métodos que são capazes de quantificar o ácido ascórbico em alimentos não fazem discriminação entre seus isômeros, o que gera imprecisão quando se quer avaliar a atividade vitamínica C. ${ }^{9,19}$ Entre os métodos incapazes de diferenciar o LAA e o DIAA está aquele indicado pela AOAC. ${ }^{21,22}$

Entre os métodos cromatográficos, a cromatografia em papel e a em camada delgada apresentam bons resultados, mas são pouco utilizadas. A cromatografia gasosa mostra boa linearidade e alta especificidade, mas envolve etapas de derivação do ácido a trimetilsilil éter. A cromatografia líquida de alta eficiência (CLAE) é a técnica mais utilizada, conferindo resultados de alta sensibilidade, especificidade e simplicidade,,$^{23,24}$ empregando colunas de fase reversa e par iônico, amina ligada, colunas amino poliméricas ou, mais frequentemente, C18. A detecção pode ser feita por técnica eletroquímica, fluorescente ou por arranjo de diodos (DAD). ${ }^{23}$

Papa-Louisi e Pascalidou ${ }^{25}$ separaram LAA, DIAA, ácido de-hidroascórbico (DHA) e ácido úrico (UA) por CLAE. Usaram DAD a $323 \mathrm{~nm}$, fase móvel composta por $0,38 \mathrm{mg} \mathrm{mL}^{-1}$ de ditiotreitol, tampão fosfato de concentração $5 \mathrm{mmol} \mathrm{L}^{-1} \mathrm{e} \mathrm{pH} \mathrm{5,0,2} \mathrm{mmol} \mathrm{L} \mathrm{me}^{-1} \mathrm{de}$ $\mathrm{Na}_{2}$ EDTA e $5 \mathrm{mmol} \mathrm{L}^{-1}$ de brometo de cetiltrimetil amônio.

O presente trabalho teve como objetivo avaliar um método de identificação e quantificação de LAA e DIAA em geleias de frutas. A repetitividade, linearidade e a recuperação foram verificadas.

\section{PARTE EXPERIMENTAL}

\section{Reagentes e padrões}

Brometo de hexadecil trimetil amônio foi obtido da Sigma; fosfato de sódio anidro, acetato de sódio e ácido meta-fosfórico foram obtidos da Merck; $\mathrm{Na}_{2}$ EDTA foi comprado da Reagen. Os padrões LAA e DIAA foram obtidos junto à Synth (São Paulo, Brasil), 99\% de pureza. A água deionizada foi produzida em deionizador Milli-Q, da Millipore.

\section{Extração das amostras}

Utilizaram-se 10 diferentes amostras de geleia de fruta: acerola com morango, laranja, rosela, acerola e goiaba, acerola e rosela, acerola e maná, acerola e maracujá, acerola, acerola e banana e, goiaba com rosela. As geleias foram obtidas do fornecedor Klaus J. G. Bouillon ME. Após o recebimento, as amostras foram divididas em pequenas porções e armazenadas sob refrigeração em frascos âmbar. A extração foi realizada próxima das analises para prevenir a degradação.

A extração foi feita com alíquotas de $0,5 \mathrm{~g}$ de geleia. A amostra foi diluída e homogeneizada em $50 \mathrm{~mL}$ de ácido meta-fosfórico $1 \%$ por $3 \mathrm{~min}$. O resultante foi centrifugado por $5 \mathrm{~min}$ a $3000 \mathrm{rpm}$, o sobrenadante foi filtrado em poros de $0,45 \mu \mathrm{m}$ e aplicado à análise por CLAE.

\section{Análise cromatográfica}

O sistema cromatográfico consistiu em um equipamento HP series 1050, com detector de arranjo de diodos acoplado (DAD).
O tratamento dos dados foi realizado no software ChromQuest. A coluna empregada foi Waters Spherisolb ODS-2 (250 x 4,6 mm). A fase móvel consistiu em 2,3 $\mathrm{mmol} \mathrm{L}^{-1}$ de brometo de hexadecil trimetil amônio, 2,5 $\mathrm{mmol} \mathrm{L}^{-1}$ de $\mathrm{Na}_{2}$ EDTA, $80 \mathrm{mmol} \mathrm{L}^{-1}$ de fosfato de sódio anidro e $20 \mathrm{mmol} \mathrm{L}^{-1}$ de acetato de sódio, ajustando a $\mathrm{pH}$ 4,5 com ácido orto-fosfórico. Foi aplicada eluição isocrática a 0,8 $\mathrm{mL} \mathrm{min}{ }^{-1}$. A detecção foi realizada a $265 \mathrm{~nm}$.

\section{Validação do método}

A seletividade do método foi avaliada utilizando-se o DAD através da comparação das respostas dos compostos em questão presentes nas amostras e também em solução padrão. Os parâmetros de comparação foram tempo de retenção, espectro de absorbância e pureza do pico, comparando os espectros no início, no ápice e no fim de cada pico. A possível presença de compostos indesejados também foi observada. A linearidade do sistema foi verificada pela construção de uma curva analítica com 10 diferentes concentrações, variando entre 10 e $200 \mathrm{mg} \mathrm{L}^{-1}$. A repetitividade foi mensurada utilizando 10 replicatas de padrões, sendo os padrões avaliados em dois níveis de concentração $\left(30\right.$ e $100 \mathrm{mg} \mathrm{L}^{-1}$ ). A repetitividade também foi verificada analisando-se por 10 replicatas a amostra de geleia com morango. Os limites de detecção (LD) e quantificação (LQ) foram obtidos a partir da curva analítica, através da divisão entre o desvio padrão (s) obtido no teste de repetitividade e o coeficiente angular da curva analítica $(S)(R=s / S)$. LD foi calculado multiplicando $R$ por 3,3 e LQ foi calculado multiplicando R por $10 .{ }^{26} \mathrm{O}$ fator de assimetria (As) foi calculado a $10 \%$ da altura da banda cromatográfica (As = b/a; onde "a" é a banda esquerda e "b" a banda direita). A resolução dos picos foi calculada com base no tempo de retenção dos picos $\left(t_{R}\right)$ e na largura da banda dos mesmos $\left(\mathrm{w}_{\mathrm{b}}\right)$ de acordo com a equação $\mathrm{Rs}=2 \mathrm{x}\left(\mathrm{t}_{\mathrm{R} 2}-\mathrm{t}_{\mathrm{R} 1}\right) /\left(\mathrm{w}_{\mathrm{b} 1}+\mathrm{w}_{\mathrm{b} 2}\right)$. O fator de separação $(\alpha)$ foi calculado de acordo com a equação $\alpha=\left(t_{R 2}-t_{m}\right) /\left(t_{R 1}-t_{m}\right)$, na qual $t_{m}$ é o tempo morto. ${ }^{27}$

\section{Taxa de recuperação}

A taxa de recuperação (REC) foi obtida através da adição de uma concentração conhecida do composto de interesse (Ca) em amostra antes da extração. A taxa é obtida pela diferença entre a concentração do composto encontrado na amostra com a fortificação (Cf) e a amostra sem a fortificação $(\mathrm{Ci})$, de acordo com a equação $\mathrm{REC}=(\mathrm{Cf}-\mathrm{Ci})$ x 100/Ca. Foram realizadas adições em dois níveis de concentração.

\section{RESULTADOS E DISCUSSÃO}

Alguns estudos identificam e quantificam ácido ascórbico em frutas e sucos, ${ }^{4-7,9,15}$ mas são raros aqueles que têm produtos de elevado grau de processamento em foco, como geleias de frutas. $\mathrm{O}$ ácido ascórbico é muito instável e deste pode ocorrer alta degradação durante a fabricação da geleia, uma vez que são necessárias etapas como cominuição, homogeneização e concentração em elevada temperatura até níveis altos de sólidos solúveis ou completo tratamento térmico (pasteurização). ${ }^{28} \mathrm{~A}$ exposição à luz, o contato com oxigênio e a alta temperatura podem degradar o ácido ascórbico.

O procedimento extrativo foi desenvolvido tendo como base o indicado como oficial pela AOAC, ${ }^{14}$ mas com algumas modificações. A primeira alteração foi no solvente: de acordo com a AOAC, a extração é realizada com ácido oxálico, mas Aldrigue ${ }^{9}$ verificou que o ácido meta-fosfórico (MPA) apresenta uma maior eficiência de extração. Outra vantagem do MPA está na sua capacidade de precipitar proteína e inativar algumas enzimas. ${ }^{9}$ Em vista destes fatos, o presente trabalho utilizou MPA 1\% como solvente de extração. 
A homogeneização da amostra em MPA foi realizada inicialmente por agitação manual, mas não foi eficiente, restando grandes partículas em solução. Para sanar esta dificuldade, a homogeneização foi feita em sonicador por $3 \mathrm{~min}$. Após esta etapa, o extrato foi centrifugado, filtrado e aplicado ao método cromatográfico.

A melhor fase móvel para separar os isômeros foi definida de acordo com revisão bibliográfica (Tabela 1). Nesta, pode ser observada a predominância da combinação de um tampão e um par iônico. $\mathrm{O}$ detector mais utilizado foi o DAD. Apenas um artigo utilizou um antioxidante artificial na fase móvel. A fase estacionária mais citada foi a com fase reversa octadecil (C18).

O presente estudo testou então o uso de uma solução tampão de fosfato de sódio e o par iônico catiônico brometo de hexadecil trimetil amônio como fase móvel. Na separação cromatográfica, o estado iônico de um composto é de fundamental importância, assim, o uso de um tampão, ácido ou base, para controle do $\mathrm{pH}$ do meio é essencial. Esta medida garante que o composto se mantenha em seu estado molecular para a retenção na fase estacionária. ${ }^{34} \mathrm{O}$ uso do par iônico na fase móvel visa modificar dinamicamente a superfície da fase estacionária, absorvendo a ela compostos iônicos hidrofóbicos, alterando os tempos de retenção. ${ }^{35}$ Este é um tipo de cromatografia mais complexo, uma vez que o equilíbrio entre o par iônico e a fase estacionária é lento, sendo mais sensível a variações de temperatura, pH e concentração do par iônico. Devido a este lento equilíbrio, a eluição por gradiente não é recomendada. ${ }^{36}$

O EDTA possui um alto potencial antioxidante e foi adicionado nas análises para prevenir a degradação dos compostos. A fase estacionária empregada foi C18. Após ensaios iniciais, a composição final e o $\mathrm{pH}$ do meio foram estabelecidos de acordo com o descrito no item Análises cromatográficas.

A validação do sistema foi realizada iniciando-se pela seletividade. Como se trata de compostos isômeros, não há diferença entre os espectros de absorção de ambos, portanto, a diferenciação dos compostos foi realizada apenas pelo tempo de retenção. Quando as amostras foram analisadas, os espectros de absorção se mantiveram semelhantes aos padrões nos tempos de retenção determinados, o que indica pureza dos picos, ou seja, ausência de interferentes em mesmo tempo de retenção dos isômeros. Em vista do exposto, o método pode ser classificado como seletivo.

A repetitividade foi testada utilizando-se soluções de 30 e 100 $\mathrm{mg} \mathrm{L}^{-1}$. Durante este procedimento, uma queda na área dos picos foi observada. Esta área é diretamente proporcional à concentração do composto, ou seja, houve degradação do mesmo. As alíquotas foram preparadas ao mesmo tempo e aplicadas no cromatográfico no decorrer das análises. A diferença entre a primeira e a última análise foi de $56 \%$. Este fato enfatiza a necessidade de se proceder às extrações e diluições tomando algumas precauções: padrões diluídos, amostras e extratos foram mantidos sob baixa temperatura, em frascos âmbar e bem vedados, até que a próxima etapa se procedesse; a extração foi feita o mais rápido possível, mantendo-se regularidade de tempo entre as diferentes amostras e, o extrato das amostras foi aplicado à técnica cromatográfica imediatamente após a extração.

Após as modificações, o desvio padrão relativo ficou abaixo de $3 \%$. A separação dos picos pode ser observada na Figura 2. Os picos apresentaram uma boa separação, com resolução de 34,68 e fator de separação de 1,21. A resolução avalia o grau de separação dos picos cromatográficos, sendo considerados valores de resolução aceitáveis aqueles acima de 1,5. O fator de separação é calculado utilizando-se apenas o tempo de retenção corrigido, sendo que valores acima de 1,0 são aceitáveis. A simetria dos picos também pode ser considerada satisfatória, à medida que foram calculados fatores de assimetria abaixo de 1,5 (1,2 para o LAA e 1,3 para o IAA). ${ }^{27} \mathrm{O}$ tempo de eluição do LAA foi 10,58 \pm 0,06 min; o DIAA eluiu após 12,32 \pm $0,07 \mathrm{~min}$. Todos os resultados foram submetidos ao teste de Grubbs, ${ }^{37}$ mas nenhum foi rejeitado. Estes tempos de eluição indicam uma boa separação, com um valor não muito próximo do início da corrida no qual geralmente se encontram muitos picos, mas também não tão longo que prejudicaria análises em sequência.

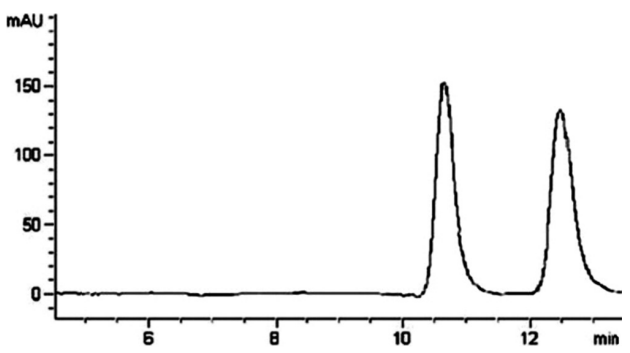

Figura 2. Cromatograma dos padrões LAA (10,58 min) e DIAA (12,32 min), ambos em concentração de $1,5 \mathrm{~g} \mathrm{~L}^{-1}$

A linearidade foi verificada construindo-se uma curva analítica. Os dois isômeros mostraram boa linearidade no intervalo avaliado, com coeficiente de correlação acima de 0,99 . Os coeficientes linear e angular do LAA foram, respectivamente, 28,5 e -33,7, Os coeficientes para o DIAA foram 29,6 e -46,2, respectivamente. Para o LAA, os

Tabela 1. Métodos de separação dos isômeros LAA e DIAA

\begin{tabular}{|c|c|c|c|c|c|c|}
\hline Composto & Amostras & Extração & Fase estacionária & Fase móvel & Detector & Ref. \\
\hline LAA e IAA & Produtos fortificados & $\begin{array}{c}\text { Tris [2-carboxietil] } \\
\text { fosfato }\end{array}$ & $\mathrm{C}_{18}$ & $\begin{array}{l}\text { Decilamina, acetonitrila, } \\
\text { acetato, } \mathrm{pH} \mathrm{5,4}\end{array}$ & DAD & 19 \\
\hline LAA e IAA & Amostras biológicas & $\mathrm{MPA}^{\mathrm{a}} 5 \%$ & $\mathrm{NH}_{2}$ & $\begin{array}{l}\text { Fosfato, água, acetonitrila, } \\
\text { ácido fosfórico }\end{array}$ & Eletroquímico & 24 \\
\hline $\begin{array}{l}\text { LAA, IAA, } \\
\text { DHA }\end{array}$ & Frutas e vegetais & $\begin{array}{c}\text { MPA } 1 \% \text { e ácido } \\
\text { oxálico } 0,5 \%, \text { pH } 2,0\end{array}$ & $\mathrm{C}_{18}$ & $\begin{array}{c}\mathrm{DTCA}^{\mathrm{b}}, \mathrm{Na}_{2} \mathrm{EDTA}, \\
\text { fosfato, acetato, pH 4,5 }\end{array}$ & UV a $247 \mathrm{~nm}$ & 29 \\
\hline $\begin{array}{l}\text { LAA, DAA, } \\
\text { DHA e UA }\end{array}$ & Amostras biológicas & $\begin{array}{c}\text { MPA } 10 \% \text { e } \\
2 \mathrm{mmol} \mathrm{L}^{-1} \text { de EDTA }\end{array}$ & $\mathrm{C}_{18}$ & $\begin{array}{l}\text { Fosfato, acetato, DTCA, } \\
\text { TCAB }^{\mathrm{c}} \text {, metanol, pH 5,4 }\end{array}$ & Colorimétrico & 30 \\
\hline LAA e IAA & $\begin{array}{l}\text { Sucos naturais, proces- } \\
\text { sados e concentrados }\end{array}$ & $\begin{array}{l}\text { Água, centrifugação e } \\
\text { filtração }\end{array}$ & $\mathrm{C}_{18}$ & Ácido acético, pH 2,5 & $\mathrm{DAD}^{\mathrm{d}}$ & 31 \\
\hline LAA e IAA & Morangos & MPA $1 \%$ & $\mathrm{C}_{18}$ & $\begin{array}{l}\text { Tampão acetato de sódio, } \\
\text { pH 4,2 }\end{array}$ & DAD & 32 \\
\hline $\mathrm{AA}$ & Suco de maçã & Filtração & $\mathrm{C}_{18}$ & $\begin{array}{l}\text { Água, acetonitrila, } \\
\text { ácido fórmico }\end{array}$ & DAD & 33 \\
\hline
\end{tabular}

á́cido meta-fosfórico; ${ }^{\mathrm{b} C l o r e t o ~ d e ~} n$-dodeciltrimetil amônio; ${ }^{\mathrm{a}}$ Brometo de tetraoctil amônio; detector de arranjo de diodos. 
limites de detecção e quantificação calculados foram 7 e $21 \mathrm{mg} \mathrm{L}^{-1}$, respectivamente. O DIAA apresentou $5 \mathrm{mg} \mathrm{L}^{-1}$ para o LD e $14 \mathrm{mg}$ $\mathrm{L}^{-1}$ para o LQ.

No final da avaliação, o método se mostrou seletivo e linear para os dois isômeros; os limites de detecção e quantificação foram adequados para as amostras em questão e, foi observada uma alta capacidade de detecção e quantificação destes compostos em geleias de frutas.

A recuperação do método foi verificada. LAA e DIAA foram adicionados em concentrações de 100 e $50 \mathrm{mg} \mathrm{L}^{-1} \mathrm{em}$ todas as amostras. Os valores obtidos pelo estudo da recuperação ficaram entre 87 e $105 \%$, demonstrando ser adequado para matrizes complexas, segundo a Associação Nacional de Vigilância Sanitária (ANVISA). ${ }^{38}$

A quantidade dos isômeros do ácido ascórbico presente nas geleias pode ser então quantificada. O isômero DIAA não foi encontrado em nenhuma das amostras analisadas; apenas o LAA foi encontrado. Na Figura 3, é apresentado o cromatograma relativo à amostra de geleia de acerola. Na Tabela 2 pode ser verificada a concentração do LAA para cada amostra, juntamente com o desvio padrão relativo. A amostra de geleia de acerola apresentou o maior desvio padrão relativo $(3,4 \%)$, já a amostra de acerola com maracujá foi aquela com menor desvio padrão $(1,12 \%)$. Estes valores são considerados adequados, ficando abaixo do valor máximo aceitável $(5 \%) .^{38}$

Tabela 2. Quantificação de ácido ascórbico nas amostras de geleia, em $100 \mathrm{~g}$, por porção de $25 \mathrm{~g}$ e em porcentagem de ingestão diária recomendada (IDR)

\begin{tabular}{lccc}
\hline Geleia & $\begin{array}{c}\text { LAA } \\
(\mathrm{mg} / 100 \mathrm{~g})\end{array}$ & $\begin{array}{c}\text { LAA } \\
(\mathrm{mg} / 25 \mathrm{~g})\end{array}$ & $\begin{array}{c}\text { LAA } \\
(\% \mathrm{IDR})\end{array}$ \\
\hline Acerola com morango & $329 \pm 6$ & $82,3 \pm 1,5$ & 137 \\
Laranja & $147 \pm 3$ & $36,7 \pm 0,8$ & 61 \\
Rosela & $\mathrm{nd}$ & $\mathrm{nd}$ & $\mathrm{nd}$ \\
Acerola com goiaba & $299 \pm 8$ & $74,7 \pm 1,5$ & 125 \\
Acerola com rosela & $320 \pm 7$ & $80,0 \pm 1,7$ & 133 \\
Acerola e maná & $281 \pm 9$ & $70,2 \pm 2,0$ & 117 \\
Acerola com maracujá & $381 \pm 4$ & $95,2 \pm 1,0$ & 159 \\
Acerola & $605 \pm 21$ & $151,2 \pm 5,2$ & 252 \\
Acerola com banana & $123 \pm 1$ & $30,7 \pm 0,2$ & 51 \\
Goiaba com rosela & $161 \pm 3$ & $40,2 \pm 0,8$ & 67 \\
\hline
\end{tabular}

nd - não detectado

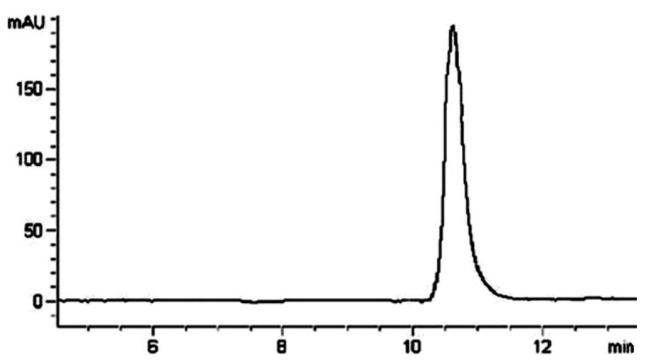

Figura 3. Cromatograma da geleia de acerola

A amostra de geleia de acerola foi aquela que apresentou maior quantidade de LAA. O composto não foi encontrado apenas na amostra de geleia de rosela. Uma comparação com as quantidades encontradas na literatura não é possível, uma vez que estudos sobre geleias são raros e poucos analisam geleias com mais de uma fruta. Uma pequena comparação foi feita utilizando dados sobre frutas, sucos e polpas para as geleias de acerola e laranja.

Araujo e colaboradores ${ }^{39}$ encontraram concentrações entre 1068 e 1836 mg de ácido ascórbico em $100 \mathrm{~g}$ de polpa de acerola congelada. Maia e colaboradores ${ }^{40}$ encontraram valores entre 573 e 594 mg de ácido ascórbico em $100 \mathrm{~mL}$ de suco de acerola. A geleia de acerola apresentou menor concentração de ácido ascórbico que a polpa, ${ }^{39}$ mas valor próximo ao do suco. ${ }^{40}$ Gokmen e colaboradores ${ }^{41}$ verificaram o referido composto em algumas frutas, incluindo laranja $(43,5 \mathrm{mg}$ em $100 \mathrm{~g}$ ). Na geleia de laranja, o ácido ascórbico foi encontrado em concentração de $147 \mathrm{mg}$ em $100 \mathrm{~g}$. A produção de geleias aplica maior grau de processos degradantes que polpas congeladas ou sucos, este fato pode justificar a diferença entre os resultados.

A ingestão diária recomendada (IDR) de vitamina $\mathrm{C}$ no Brasil e nos Estados Unidos é de $60 \mathrm{mg}$ para um adulto.. ${ }^{42,43}$ Uma porção de geleia representa $25 \mathrm{mg}$ (uma colher de sopa). Seis das geleias quantificadas apresentaram quantidades acima da IDR para uma porção. A ingestão de um pouco mais de uma porção das geleias de laranja, acerola com banana e goiaba com rosela é necessária para se alcançar a IDR, não necessitando de duas porções.

\section{CONCLUSÃO}

O método proposto e avaliado identificou e quantificou os isômeros ácido L-ascórbico e ácido D-iso-ascórbico em geleias de frutas. O método demonstrou ser seletivo, linear, repetitivo e com elevado grau de recuperação. O DIAA não foi encontrado nas amostras. O LAA foi encontrado nas amostras em concentrações de até $605 \mathrm{mg}$ em $100 \mathrm{~g}$ de amostras. Seis amostras apresentaram valores acima da quantidade diária recomendada, três mostraram valores acima da IDR para até duas porções e em apenas uma geleia não foi encontrado o ácido ascórbico.

\section{AGRADECIMENTOS}

À CAPES pelo financiamento dos estudos na Universidade Estadual de Campinas.

\section{REFERÊNCIAS}

1. Barata-Soares, A. D.; Gomez, M. L.; Mesquita, C. H.; Lajojo, F. M.; Braz. J. Plant Physiol. 2004, 16, 147.

2. Hancock, R. D.; Recent Pat. on Food, Nutr. Agric. 2009, 1, 39.

3. Arrigoni, O.; Tullio, M.; Biochim. Biophys. Acta, Gen. Subj. 2002, 1569, 1 .

4. Valente, A.; Albuquerque, T. G.; Sanches-Silva, A.; Costa, H. S.; Food Res. Int. 2011, 44, 2237.

5. Wolkoff, D. B.; Tese de Doutorado, Universidade Estadual de Campinas, Brasil, 2004.

6. Lopez, A.; Montano, A.; Garcia, P.; Garrido, A.; In. J. Food Sci. Technol. 2005, 11, 199.

7. Nojavam, S.; Khalilian, F.; Kiaie, F. M.; Rahimi, A.; Arabanian, A.; Chalavi, S.; J. Food Compos. Anal. 2008, 21, 300.

8. Nachtigall, A. M.; Granada, G. G.; Zambiazi, R. C.; Resumos do XVIIICongresso Brasileiro de Ciência e Tecnologia de Alimentos, Porto Alegre, Brasil, 2002.

9. Aldrigue, M. L.; Tese de Doutorado, Universidade Estadual de Campinas, Brasil, 1999.

10. Davey, M. W.; Montagu, M. V.; Inze, D.; Sanmartin, M.; Kanellis, A.; Smirnoff, N.; Benzie, I. J. J.; Favell, D.; Fletcher, J.; J. Sci. Food Agr. 2000, 80,825 .

11. Davey, M. W.; Bauw, G.; Montagu, M. V.; Anal. Biochem. 1996, 239, 8.

12. Gabas, A. L.; Telis-Romero, J.; Menegalli, F. C.; Ciênc. Tecnol. Aliment. 2003, 23, 66.

13. Emadi-Konjin, P.; Verjee Z.; Levin, A. V.; Adeli, K.; Clin. Biochem. 2005, 38,450 .

14. Association of Official Analytical Chemists; Official methods of analysis of AOAC international, 16 ${ }^{\text {th }}$ ed., AOAC: Gaitherburg, 1997. 
15. Raghu, V.; Platel, K.; Srinivasan, K.; J. Food Comp. Anal. 2007, 20,529.

16. Wang, Y.; Mackenzie, B.; Tsukaguchi, H.; Weremowicz, S.; Morton, C. C.; Hediger, M. A.; Biochem. Biophys. Res. Commun. 2000, 267, 488.

17. Hidiroglou, N.; Madere, R.; L'Abbé, M. R.; J. Nutr. Biochem. 1997, 8, 13.

18. Fidler, M. C.; Davidsson, L.; Zeder, C.; Hurrel, R. F.; Am. J. Clin. Nutr. 2004, 79, 99.

19. Fontannaz, P.; Kilinic, T.; Heudi, O.; Food Chem. 2006, 94, 626.

20. Sadecka, J.; Polonsky, J.; Eur. Food Res. Technol. 2001, $212,511$.

21. Barros, A. I.; Silva, A. P.; Gonçalves, B.; Nunes, F. M.; Anal. Bioanal. Chem. 2010, 396, 1863.

22. Hugues, R. E.; Hurley, R. J.; Br. J. Nutr.1969, 23, 211.

23. Sanchez-Mata, M. C.; Camara-Hurtado, M.; Diez-Marques, C.; Esperanza, M.; Torija-Isasa.; Eur. Food Res. Technol. 2000, 210, 220.

24. Margohs, S. A.; Schapira, R. M.; J. Chromatogr., B: Anal. Technol. Biomed Life Sci. 1997, 690, 25.

25. Papa-Louisi, A.; Pascalidou, S.; Anal. Biochem.1998, 263, 176.

26. Ribani, M.; Bottoli, C. B. G.; Collins, C. H.; Jardim, I. C. S. F.; Mello, L. F. C.; Quim. Nova 2004, 27, 771.

27. Snyder, L. R.; Kirkland, J. J.; Glajch, J. L.; Practical HPLC Method Development, $2^{\text {nd }}$ ed., Wiley: New York, 1997.

28. Baker, R. A.; Berry, N.; Hui, Y. H.; Barret, D. M.; Fruit Preserves and Jams in Processing Fruit: Science and Technology, $2^{\text {nd }}$ ed., CRC Press: New York, 2005.

29. Kall, M. A.; Andersen, C.; J. Chromatogr., B: Anal. Technol. Biomed Life Sci. 1999, 730, 101.

30. Lykkesfeldt, J.; Anal. Biochem. 2000, 282, 89.
31. Furusawa, N.; Food Control. 2001, 12, 27.

32. Cordenunsi, B. R.; Nascimento, J. R.; Genovese, M. I.; Lajolo, F. M.; J. Agric. Food Chem. 2002, 50, 2581.

33. Tikekar, R. V.; Anantheswaran, R. C.; LaBorde, L. F.; J. Food Sci. 2011, 76, H62.

34. Rosa, J. S.; Godoy, R. L. O.; Oiano Neto, J.; Campos, R. S.; Matta, V. M.; Freire, C. A.; Silva., A. S.; Souza, R. S.; Ciênc. Tecnol. Aliment. 2007, 27, 837.

35. Cecchi, T.; Crit. Rev. Anal. Chem. 2009, 38, 161

36. Bastos, C. A.; Tese de Doutorado, Universidade Federal de Juiz de Fora, Brasil, 2008.

37. Grubbs, F. E.; Technometrics 1969, 11, 1 .

38. Agência Nacional de Vigilância Sanitária (ANVISA); Guia para Validação de Métodos Analíticos e Bioanáliticos, RE no 899, 29/5/2003.

39. Araújo, P. G.; Figueiredo, R. W.; Alves, R. E.; Maria, G. A.; Paiva, J. R.; Ciênc. Tecnol. Aliment. 2007, 27, 104.

40. Maia, G. A.; Sousa, P. H.; Santos, G. M.; Silva, D. S.; Fernandes, A. G.; Prado, G. M.; Ciênc. Tecnol. Aliment. 2007, 27, 787.

41. Gokmen, V.; Kahraman, N.; Demir, N.; Acar, J.; J. Chromatogr., A 2000, $309,881$.

42. Subcommittee on the $10^{\text {th }}$ edition of the RDAs, Food and Nutrition Board, Commission on Life Sciences, National Research Council; Recomended Dietary Allowances, National Academy Press: Washington, DC, 1989, vol. 10, p. 115.

43. Agência Nacional de Vigilância Sanitária (ANVISA); Portaria SVS/ MS n³3, Tabelas de Ingestão Diária Recomendada (IDR). s.l. : Diário Oficial da União, 16 de janeiro de 1998. 